\title{
EXPLICIT FORMULAE FOR TWO-BRIDGE KNOT POLYNOMIALS
}

\author{
SHINJI FUKUHARA
}

(Received 1 August 2001; revised 5 May 2003)

\author{
Communicated by S. Gadde
}

\begin{abstract}
A two-bridge knot (or link) can be characterized by the so-called Schubert normal form $K_{p, q}$ where $p$ and $q$ are positive coprime integers. Associated to $K_{p, q}$ there are the Conway polynomial $\nabla_{K_{p, q}}(z)$ and the normalized Alexander polynomial $\Delta_{K_{p . q}}(t)$. However, it has been open problem how $\nabla_{K_{p . q}}(z)$ and $\Delta_{K_{p, q}}(t)$ are expressed in terms of $p$ and $q$. In this note, we will give explicit formulae for the Conway polynomials and the normalized Alexander polynomials in the case of two-bridge knots and links. This is done using elementary number theoretical functions in $p$ and $q$.
\end{abstract}

2000 Mathematics subject classification: primary 57M25; secondary 1 IL03.

Keywords and phrases: two-bridge knot, Alexander polynomial, Conway polynomial.

\section{Introduction and statement of results}

Let $K$ be a knot (or link) in $\mathbb{R}^{3}$, and let $\Delta_{K}(t)$ be the normalized Alexander polynomial (reduced when $K$ is a link) for $K$. There is also the Conway polynomial $\nabla_{K}(z)$ of $K$ in the variable $z$. The Conway polynomial is related to the Alexander polynomial $\Delta_{K}(t)$ by the equation:

$$
\nabla_{K}\left(t^{-1 / 2}-t^{1 / 2}\right)=\Delta_{K}(t)
$$

A two-bridge knot (or link) can be characterized by the so-called Schubert normal form $K_{p, q}$ where $p$ and $q$ are positive coprime integers. (Figure 1 illustrates the diagram $K_{5,3}$.) The reader is referred to Burde-Zieschang [3] or Kawauchi [9] for more detailed description of $K_{p, q}$. It is known that $K_{p, q}$ is a knot if $p$ is odd, respectively, a two-component link if $p$ is even. (Figure 2 illustrates the two-component link $K_{4,1}$.)

The author wishes to thank Professor N. Yui and the referee for their helpful advice.

(C) 2005 Australian Mathematical Society 1446-7887/05 $\$$ A2.00+0.00 


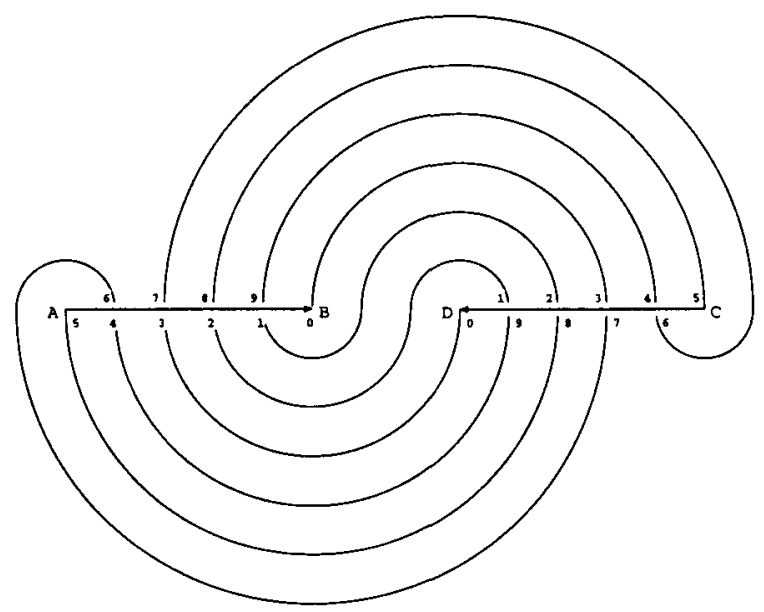

FIGURE 1. The knot $K_{5,3}$.

Throughout this article, we use the notation $K_{p, q}$ for an oriented knot (or link) which are represented by the diagram $K_{p, q}$. Our main purpose of this note is to express explicitly the Alexander polynomials and the Conway polynomials of $K_{p, q}$ in terms of $p$ and $q$. Explicit descriptions of these polynomials have been sought after by many knot theorists (see $[7,8]$ for algorithms of calculating the Alexander polynomials of $K_{p, q}$ ). However, the problem has been rather intractable. One motivation stems from the same problem for a torus knot $T_{p, q}$ of type $(p, q)$. For $T_{p, q}$, the Alexander polynomial has a beautiful simple expression given as follows:

$$
\Delta_{T_{p, q}}(t)=\frac{\left(t^{-1 / 2}-t^{1 / 2}\right)\left(t^{-p q / 2}-t^{p q / 2}\right)}{\left(t^{-p / 2}-t^{p / 2}\right)\left(t^{-q / 2}-t^{q / 2}\right)}
$$

Unfortunately, in the case of $K_{p, q}$, one cannot hope to obtain such a nice expression for the Alexander polynomial.

One of the reasons is the fact that $K_{p, q}$ is equivalent to $K_{p, q+2 p}$ while $T_{p, q}$ is not equivalent to $T_{p, q+2 p}$ in general (this means that $\Delta_{K_{p, q+2 p}}(t)$ should coincide with $\Delta_{K_{p, q}}(t)$ while $\Delta_{T_{p, q+2 p}}(t)$ and $\Delta_{T_{p, q}}(t)$ can be independent).

In order to obtain explicit expressions for $\Delta_{K_{p, q}}(t)$, we should look for a function $f(p, q)$ in $p$ and $q$ satisfying the following equation:

$$
f(p, q)=f(p, q+2 p)
$$

It should be remarked that such a function is related to Dedekind symbols introduced in [6]. This function should be of great interest from a number theoretical view point, see, for example, $[1,10]$. 


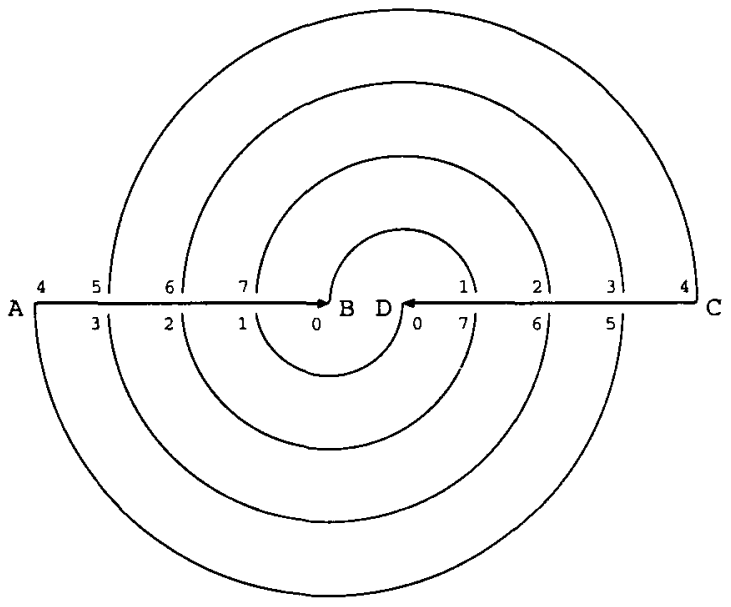

FIGURE 2. The two-component link $K_{4,1}$.

Hence we introduce functions $\varepsilon_{i}, \mu$ and $v_{k}$ as follows. First let us fix notation; by $[x]$, we denote the greatest integer not exceeding $x \in \mathbb{R}$.

DEFINITION 1.1. For integers $i$ and $k$, we define $\varepsilon_{i}(p, q), \mu(p, q)$ and $\nu_{k}(p, q)$ by

$$
\varepsilon_{i}(p, q):=(-1)^{[i q / p]}, \quad \mu(p, q):=\sum_{i=1}^{p-1} \varepsilon_{i} \quad \text { and } \quad v_{k}(p, q):=1+\sum_{i=1}^{p-1} \varepsilon_{k+i} .
$$

It can be checked easily that these functions satisfy (1.2). We also know that $v_{0}(p, q)=\mu(p, q)+1$.

In what follows, we write simply $\varepsilon_{i}, \mu$ and $\nu_{k}$ instead of $\varepsilon_{i}(p, q), \mu(p, q)$ and $\nu_{k}(p, q)$ to ease the notation. Though the reader should keep in mind that $\varepsilon_{i}, \mu$ and $\nu_{k}$ do depend on $p$ and $q$.

Furthermore, whenever we are dealing with a two-bridge knot $K_{p, q}$, we may assume without loss of generality that $q$ is odd (as any two-bridge knot can be reduced to such a case).

We can now give an explicit formula for (reduced) normalized Alexander polynomial $\Delta_{K_{p, q}}$. The results are formulated as follows:

THEOREM 1.2. (1) For a two-bridge knot $K_{p, q}$,

$$
\Delta_{K_{p . q}}(t)=\frac{1}{2}\left(t^{-\mu / 2}+t^{\mu / 2}\right)-\frac{1}{4}\left(t^{-1 / 2}-t^{1 / 2}\right) \sum_{k=1}^{p-1}(-1)^{k} \varepsilon_{k}\left(t^{-\nu_{k} / 2}-t^{\nu_{k} / 2}\right) .
$$


(2) For a two-bridge link $K_{p, q}$,

$$
\Delta_{K_{p . q}}(t)=\frac{1}{2}\left(t^{-\mu / 2}-t^{\mu / 2}\right)-\frac{1}{4}\left(t^{-1 / 2}-t^{1 / 2}\right) \sum_{k=1}^{p-1}(-1)^{k} \varepsilon_{k}\left(t^{-v_{k} / 2}+t^{v_{k} / 2}\right) .
$$

Next we will consider the Conway polynomial of $K_{p, q}$. Note that the Alexander polynomial can be derived easily from the Conway polynomial by substituting $t^{-1 / 2}-t^{1 / 2}$ for $z$. However, the other way around is not so easy. Thus, by the aid of the Lucas polynomials, we will transform the Alexander polynomial to the Conway polynomial (the definition of the Lucas polynomials will be given in Section 5). Our result is formulated as follows:

THEOREM 1.3. (1) For a two-bridge knot $K_{p, q}$,

$$
\begin{aligned}
\nabla_{K_{p, q}}(z)= & \frac{1}{2} \sum_{j=0}^{|\mu| / 2} \frac{|\mu|}{|\mu| / 2+j}\left(\begin{array}{c}
|\mu| / 2+j \\
|\mu| / 2-j
\end{array}\right) z^{2 j} \\
& -\frac{1}{4} \sum_{k=1}^{p-1}(-1)^{k} \varepsilon_{k} \sum_{j=0}^{\left(\left|\nu_{k}\right|-1\right) / 2} \frac{\nu_{k}}{\left|v_{k}\right| / 2+1 / 2+j}\left(\begin{array}{c}
\left|\nu_{k}\right| / 2+1 / 2+j \\
\left|v_{k}\right| / 2-1 / 2-j
\end{array}\right) z^{2 j+2} .
\end{aligned}
$$

(2) For a two-bridge link $K_{p, q}$,

$$
\begin{aligned}
\nabla_{K_{p . q}}(z)= & \frac{1}{2} \sum_{j=0}^{(|\mu|-1) / 2} \frac{\mu}{|\mu| / 2+1 / 2+j}\left(\begin{array}{l}
|\mu| / 2+1 / 2+j \\
|\mu| / 2-1 / 2-j
\end{array}\right) z^{2 j+1} \\
& -\frac{1}{4} \sum_{k=1}^{p-1}(-1)^{k} \varepsilon_{k} \sum_{j=0}^{\left|\nu_{k}\right| / 2} \frac{\left|\nu_{k}\right|}{\left|\nu_{k}\right| / 2+j}\left(\begin{array}{c}
\left|\nu_{k}\right| / 2+j \\
\left|\nu_{k}\right| / 2-j
\end{array}\right) z^{2 j+1}
\end{aligned}
$$

Here we regard

$$
\frac{|\mu|}{|\mu| / 2+j}\left(\begin{array}{l}
|\mu| / 2+j \\
|\mu| / 2-j
\end{array}\right)=2 \quad\left(\text { respectively } \frac{\left|v_{k}\right|}{\left|v_{k}\right| / 2+j}\left(\begin{array}{l}
\left|v_{k}\right| / 2+j \\
\left|v_{k}\right| / 2-j
\end{array}\right)=2\right)
$$

when $|\mu|=j=0$ (respectively $\left|v_{k}\right|=j=0$ ).

Finally we present an alternative formula for $\nabla_{K_{p, q}}(z)$ by describing its coefficients explicitly. For a knot (or link) $K$, let $a_{j}(K)$ denote the $j$ th coefficient of the Conway polynomial $\nabla_{K}(z)$, that is,

$$
\nabla_{K}(z)=\sum_{j=0} a_{j}(K) z^{j}
$$

Then $a_{j}\left(\nabla_{K_{p, q}}\right)$ is expressed as follows: 
THEOREM 1.4. (1) For a two-bridge knot $K_{p, q}$,

$$
\begin{aligned}
a_{0}\left(K_{p, q}\right)= & 1, \quad a_{2 j+1}\left(K_{p, q}\right)=0 \quad(j \geq 0), \\
a_{2 j+2}\left(K_{p, q}\right)= & \frac{1}{2^{2 j+2}(2 j+2) !}\left[\prod_{m=0}^{j}\left(\mu^{2}-4 m^{2}\right)\right. \\
& \left.-(2 j+2) \sum_{k=1}^{p-1}(-1)^{k} \varepsilon_{k} \nu_{k} \prod_{m=1}^{j}\left\{v_{k}^{2}-(2 m-1)^{2}\right\}\right] \quad(j \geq 0) .
\end{aligned}
$$

(2) For a two-bridge link $K_{p, q}$,

$$
\begin{aligned}
a_{2 j}\left(K_{p, q}\right)= & 0 \quad(j \geq 0), \\
a_{2 j+1}\left(K_{p, q}\right)= & \frac{1}{2^{2 j+1}(2 j+1) !}\left[\mu \prod_{m=1}^{j}\left\{\mu^{2}-(2 m-1)^{2}\right\}\right. \\
& \left.-(2 j+1) \sum_{k=1}^{p-1}(-1)^{k} \varepsilon_{k} \prod_{m=0}^{j-1}\left(v_{k}^{2}-4 m^{2}\right)\right] \quad(j \geq 0) .
\end{aligned}
$$

Here we regard

$$
\prod_{m=1}^{j}\left\{v_{k}^{2}-(2 m-1)^{2}\right\}=\prod_{m=1}^{j}\left\{\mu^{2}-(2 m-1)^{2}\right\}=\prod_{m=0}^{j-1}\left(v_{k}^{2}-4 m^{2}\right)=1
$$

when $j=0$.

\section{Some properties of numbers $\varepsilon_{i}, \mu$ and $v_{k}$}

In order to prove the theorems we need several properties of numbers $\varepsilon_{i}, \mu$ and $\nu_{k}$.

LEMMA 2.1. (1) For $i$ such that $0<i<p, \varepsilon_{p-i}=\varepsilon_{i}$.

(2) For $i$ such that $0<i<p, \varepsilon_{p+i}=-\varepsilon_{i}$.

(3) $\varepsilon_{p}=-1$.

Proof. We recall the assumption that $q$ is odd (namely $(-1)^{q}=-1$ ). We also note that identities $[-x]=-[x]-1$ and $[n+x]=n+[x]$ hold for $x \in \mathbb{R} \backslash \mathbb{Z}$ and $n \in \mathbb{Z}$. Then we have
(1) $\varepsilon_{p-i}=(-1)^{[(p-i) q / p]}=(-1)^{q+[-i q / p]}=(-1)^{q-[i q / p]-1}=(-1)^{[i q / p]}=\varepsilon_{i}$;
(2) $\varepsilon_{p+i}=(-1)^{[(p+i) q / p]}=(-1)^{q+[i q / p]}=-(-1)^{[i q / p]}=-\varepsilon_{i}$;
(3) $\varepsilon_{p}=(-1)^{[p q / p]}=(-1)^{q}=-1$. 
The following identities can derived using Lemma 2.1.

LEMMA 2.2. (1) For $k$ such that $0<k<p, \sum_{i=1}^{k-1} \varepsilon_{i}=\mu-\sum_{i=1}^{p-k} \varepsilon_{i}$.

(2) For $k$ such that $0<k<p$,

$$
-\frac{\mu}{2}+\sum_{i=1}^{k-1} \varepsilon_{i}+\frac{\varepsilon_{k}-1}{2}=-\frac{\nu_{k}}{2}-\frac{1}{2} .
$$

PRoOF. (1) By Lemma 2.1 (1), we have

$$
\sum_{i=1}^{k-1} \varepsilon_{i}=\mu-\sum_{i=1}^{p-1} \varepsilon_{i}+\sum_{i=1}^{k-1} \varepsilon_{i}=\mu-\sum_{i=k}^{p-1} \varepsilon_{i}=\mu-\sum_{i=1}^{p-k} \varepsilon_{p-i}=\mu-\sum_{i=1}^{p-k} \varepsilon_{i} .
$$

(2) Next applying (2) and (3) of Lemma 2.1, we obtain

$$
\begin{aligned}
-\frac{\mu}{2} & +\sum_{i=1}^{k-1} \varepsilon_{i}+\frac{\varepsilon_{k}-1}{2} \\
& =-\frac{1}{2} \sum_{i=1}^{p-1} \varepsilon_{i}+\sum_{i=1}^{k-1} \varepsilon_{i}+\frac{\varepsilon_{k}-1}{2}=-\frac{1}{2} \sum_{i=k+1}^{p-1} \varepsilon_{i}+\frac{1}{2} \sum_{i=1}^{k-1} \varepsilon_{i}-\frac{1}{2} \\
& =-\frac{1}{2} \sum_{i=k+1}^{p-1} \varepsilon_{i}-\frac{1}{2} \sum_{i=1}^{k-1} \varepsilon_{p+i}-\frac{1}{2} \varepsilon_{p}-1 \quad \text { (by Lemma 2.1 (2)-(3)) } \\
& =-\frac{1}{2} \sum_{i=1}^{p-1} \varepsilon_{k+i}-1=-\frac{\nu_{k}}{2}-\frac{1}{2} .
\end{aligned}
$$

\section{The Wirtinger presentation for the knot (or link) group of $K_{p, q}$}

In the next section we will obtain the Alexander polynomial $\Delta_{K_{p, q}}$ applying Fox calculus. To do so we first study the Wirtinger presentation for the knot (or link) group of $K_{p, q}$. Let

$$
L_{1}:=S_{2}^{\varepsilon_{1}} S_{1}^{\varepsilon_{2}} \cdots S_{2}^{\varepsilon_{p-2}} S_{1}^{\varepsilon_{p-1}} \quad \text { and } \quad L_{2}:=S_{2}^{\varepsilon_{1}} S_{1}^{\varepsilon_{2}} \cdots S_{1}^{\varepsilon_{p-2}} S_{2}^{\varepsilon_{p-1}}
$$

be words in $S_{1}$ and $S_{2}$. Put $R_{1}:=S_{1} L_{1} S_{2}^{-1} L_{1}^{-1}$ and $R_{2}:=S_{1} L_{2} S_{1}^{-1} L_{2}^{-1}$. From the diagram $K_{p, q}$ like Figure 1 or Figure 2, the reader can easily read off the following Wirtinger presentation (also refer to [3, page 208] and note that our relators $R_{1}$ and $R_{2}$ are conjugate elements of their relators).

When $p$ is odd, the knot group of $K_{p, q}$ is presented by $G_{1}:=\left\langle S_{1}, S_{2} \mid R_{1}\right\rangle$. When $p$ is even, the link group of $K_{p, q}$ is presented by $G_{2}:=\left\langle S_{1}, S_{2} \mid R_{2}\right\rangle$. 


\section{The Alexander polynomial of a two-bridge $\operatorname{knot} K_{p, q}$}

In this section we will obtain the normalized Alexander polynomial of a two-bridge knot $K_{p, q}$ from the Wirtinger presentation, applying Fox calculus. From here to Section 5, we assume $p$ is odd so that $K_{p, q}$ is a knot. We use the following notation: let $\alpha: \mathbb{Z} F\left(S_{1}, S_{2}\right) \rightarrow \mathbb{Z}\left[t, t^{-1}\right]$ be an abelianization map such that $\alpha\left(S_{1}\right)=t, \alpha\left(S_{2}\right)=t$. We also use the symbol $(u)^{\alpha}$ instead of $\alpha(u)$.

Since $G_{1}=\left\langle S_{1}, S_{2} \mid R_{1}\right\rangle$ is the Wirtinger presentation of $K_{p, q}$, we know both $\left(\partial R_{1} / \partial S_{1}\right)^{\alpha}$ and $\left(\partial R_{1} / \partial S_{2}\right)^{\alpha}$ are Alexander polynomials. This follows from the theory of Fox calculus (the reader can refer to [4] or [3, Chapter 9] for Alexander polynomials and Fox calculus).

We introduce symbols $D_{1}(t)$ and $D_{2}(t)$ :

$$
D_{1}(t):=\left(\frac{\partial R_{1}}{\partial S_{1}}\right)^{\alpha} \text { and } D_{2}(t):=\left(\frac{\partial R_{1}}{\partial S_{2}}\right)^{\alpha}
$$

to emphasize that they are (Laurent) polynomials in $t$. Then both $D_{1}(t)$ and $D_{2}(t)$ are Alexander polynomials for $K_{p, q}$ (not normalized!). In the following lemma we obtain explicit formulae for $D_{1}(t)$ and $D_{2}(t)$.

$$
\begin{aligned}
& \text { LEMMA 4.1. (1) } D_{1}(t)=1+(t-1) \sum_{k=1, k \text { even }}^{p-1} \varepsilon_{k} t^{\sum_{i=1}^{k-1} \varepsilon_{i}+\left(\varepsilon_{k}-1\right) / 2} \text {. } \\
& \text { (2) } D_{2}(t)=-t^{\mu}+(t-1) \sum_{k=1, k \text { odd }}^{p-1} \varepsilon_{k} t^{\sum_{i=1}^{k-1} \varepsilon_{i}+\left(\varepsilon_{k}-1\right) / 2} .
\end{aligned}
$$

PROOF. By the definition of the free derivative $\partial / \partial S_{1}$, we have

$$
\begin{aligned}
\left(\frac{\partial L_{1}}{\partial S_{1}}\right)^{\alpha} & =\left(\frac{\partial S_{2}^{\varepsilon_{1}} S_{1}^{\varepsilon_{2}} \cdots S_{2}^{\varepsilon_{p-2}} S_{1}^{\varepsilon_{p-1}}}{\partial S_{1}}\right)^{\alpha} \\
& =\left(\sum_{k=1, k \text { even }}^{p-1} \varepsilon_{k} S_{2}^{\varepsilon_{1}} S_{1}^{\varepsilon_{2}} \cdots S_{1}^{\left(\varepsilon_{k}-1\right) / 2}\right)^{\alpha}=\sum_{k=1, k \text { even }}^{p-1} \varepsilon_{k} t^{\sum_{i=1}^{k-1} \varepsilon_{i}+\left(\varepsilon_{k}-1\right) / 2} .
\end{aligned}
$$

Similarly we have

$$
\left(\frac{\partial L_{1}}{\partial S_{2}}\right)^{\alpha}=\sum_{k=1, k \text { odd }}^{p-1} \varepsilon_{k} t^{\sum_{i=1}^{k-1} \varepsilon_{i}+\left(\varepsilon_{k}-1\right) / 2}
$$

Next we calculate $D_{1}(t)=\left(\partial R_{1} / \partial S_{1}\right)^{\alpha}$ as follows:

$$
D_{1}(t)=\left(\frac{\partial S_{1} L_{1} S_{2}^{-1} L_{1}^{-1}}{\partial S_{1}}\right)^{\alpha}=1+\left(S_{1} \frac{\partial L_{1}}{\partial S_{1}}\right)^{\alpha}-\left(S_{1} L_{1} S_{2}^{-1} L_{1}^{-1} \frac{\partial L_{1}}{\partial S_{1}}\right)^{\alpha}
$$




$$
=1+(t-1)\left(\frac{\partial L_{1}}{\partial S_{1}}\right)^{\alpha}=1+(t-1) \sum_{k=1, k \text { even }}^{p-1} \varepsilon_{k} t^{\sum_{i=1}^{k-1} \varepsilon_{i}+\left(\varepsilon_{k}-1\right) / 2} .
$$

Similarly, we have

$$
\begin{aligned}
D_{2}(t) & =\left(\frac{\partial S_{1} L_{1} S_{2}^{-1} L_{1}^{-1}}{\partial S_{2}}\right)^{\alpha}=\left(S_{1} \frac{\partial L_{1}}{\partial S_{2}}\right)^{\alpha}-\left(S_{1} L_{1} S_{2}^{-1}\right)^{\alpha}-\left(S_{1} L_{1} S_{2}^{-1} L_{1}^{-1} \frac{\partial L_{1}}{\partial S_{2}}\right)^{\alpha} \\
& =-t^{\sum_{i=1}^{p-1} \varepsilon_{i}}+(t-1)\left(\frac{\partial L_{1}}{\partial S_{2}}\right)^{\alpha}=-t^{\mu}+(t-1) \sum_{k=1, k \text { odd }}^{p-1} \varepsilon_{k} t^{\sum_{i=1}^{k-1} \varepsilon_{i}+\left(\varepsilon_{k}-1\right) / 2}
\end{aligned}
$$

This completes the proof.

Lemma 4.1 gives a description for the non-normalized Alexander polynomials for $K_{p, q}$. To find the normalized Alexander polynomials for $K_{p, q}$, we need the following two lemmas.

LEMMA 4.2. $D_{1}(t)=-D_{2}(t)$.

PROOF. From the fundamental formula for free calculus ([2, Proposition 3.4]), we have

$$
\frac{\partial R_{1}}{\partial S_{1}}\left(S_{1}-1\right)+\frac{\partial R_{1}}{\partial S_{2}}\left(S_{2}-1\right)=R_{1}-1 .
$$

Sending it by the abelianization map, we have

$$
\left(\frac{\partial R_{1}}{\partial S_{1}}\right)^{\alpha}(t-1)+\left(\frac{\partial R_{1}}{\partial S_{2}}\right)^{\alpha}(t-1)=0
$$

where we used $\alpha\left(R_{1}\right)=1$.

Since $\mathbb{Z}\left[t, t^{-1}\right]$ is an integral domain, we can cancel $t-1$ in (4.1). Hence we have proved the lemma.

We study another relation between $D_{1}(t)$ and $D_{2}(t)$.

LEMMA 4.3. $D_{1}(t)=-t^{\mu} D_{2}\left(t^{-1}\right)$.

ProOF. By Lemma 2.1 and Lemma 2.2, we have

$$
\begin{aligned}
\sum_{i=1}^{k-1} \varepsilon_{i}+\left(\varepsilon_{k}-1\right) / 2 & =\mu-\sum_{i=1}^{p-k} \varepsilon_{i}+\left(\varepsilon_{k}-1\right) / 2 \quad(\text { by Lemma } 2.2(1)) \\
& =\mu-\sum_{i=1}^{p-k} \varepsilon_{i}+\left(\varepsilon_{p-k}-1\right) / 2 \quad(\text { by Lemma } 2.1(1)) .
\end{aligned}
$$


Using these identities, we obtain

$$
\begin{aligned}
D_{1}(t) & =1+(t-1) \sum_{k=1, k \text { even }}^{p-1} \varepsilon_{k} t_{i=1}^{k-1} \varepsilon_{i}+\left(\varepsilon_{k}-1\right) / 2 \\
& =1+(t-1) \sum_{k=1, k \text { even }}^{p-1} \varepsilon_{p-k} t^{\mu-\sum_{i=1}^{p-k} \varepsilon_{i}+\left(\varepsilon_{p-k}-1\right) / 2} \\
& =1+(t-1) \sum_{k=1, k \text { odd }}^{p-1} \varepsilon_{k} t^{\mu-\sum_{i=1}^{k} \varepsilon_{i}+\left(\varepsilon_{k}-1\right) / 2} \quad \text { (by Lemma 2.1 (1)) } \\
& =t^{\mu}\left\{t^{-\mu}+\left(1-t^{-1}\right) \sum_{k=1, k \text { odd }}^{p-1} \varepsilon_{k} t^{-\sum_{i=1}^{k-1} \varepsilon_{i}-\left(\varepsilon_{k}-1\right) / 2}\right\} \\
& =t^{\mu}\left\{-D_{2}\left(t^{-1}\right)\right\}=-t^{\mu} D_{2}\left(t^{-1}\right) .
\end{aligned}
$$

This completes the proof.

Now we are ready to get the normalized Alexander polynomial for $K_{p, q}$. Set

$$
D(t):=t^{-\mu / 2} D_{1}(t)
$$

LEMMA 4.4. $D(t)$ is the normalized Alexander polynomial. Namely, $D(t)$ satisfies $D\left(t^{-1}\right)=D(t)$ and $D(1)=1$.

PROOF. We know that $D_{1}(t)=-t^{\mu} D_{2}\left(t^{-1}\right)$ (Lemma 4.3). This implies $D_{1}\left(t^{-1}\right)=$ $-t^{-\mu} D_{2}(t)$. We also know $D_{1}(t)=-D_{2}(t)$ by Lemma 4.2. Using these identities, we have

$$
D\left(t^{-1}\right)=t^{\mu / 2} D_{1}\left(t^{-1}\right)=t^{\mu / 2}\left\{-t^{-\mu} D_{2}(t)\right\}=t^{-\mu / 2} D_{1}(t)=D(t) .
$$

Furthermore, we have $D(1)=D_{1}(1)=1$ which completes the proof.

Lemma 4.4 shows that $D(t)$ coincides with $\Delta_{K_{p, q}}$. This is a nice result, but short of our final goal. We wish to have an expression for $\Delta_{K_{p, q}}$ with more symmetry in $t$ and $t^{-1}$. We will proceed with calculation to find a more symmetric expression of $\Delta_{K_{p, q}}$.

\section{LEMMA 4.5 .}

$$
2 D(t)=t^{-\mu / 2}+t^{\mu / 2}+(t-1) \sum_{k=1}^{p-1}(-1)^{k} \varepsilon_{k} t^{-\mu / 2+\sum_{i=1}^{k-1} \varepsilon_{i}+\left(\varepsilon_{k}-1\right) / 2} .
$$


ProOF. We have

$$
\begin{aligned}
2 D(t)= & t^{-\mu / 2} D_{1}(t)-t^{-\mu / 2} D_{2}(t) \quad\left(\text { since } D_{1}(t)=-D_{2}(t)\right) \\
= & t^{-\mu / 2}+(t-1) \sum_{k=1, k \text { even }}^{p-1} \varepsilon_{k} t^{-\mu / 2+\sum_{i=1}^{k-1} \varepsilon_{i}+\left(\varepsilon_{k}-1\right) / 2} \\
& +t^{\mu / 2}-(t-1) \sum_{k=1, k \text { odd }}^{p-1} \varepsilon_{k} t^{-\mu / 2+\sum_{i=1}^{k-1} \varepsilon_{i}+\left(\varepsilon_{k}-1\right) / 2} \quad \text { (by Lemma 4.1) } \\
= & t^{-\mu / 2}+t^{\mu / 2}+(t-1) \sum_{k=1}^{p-1}(-1)^{k} \varepsilon_{k} t^{-\mu / 2+\sum_{i=1}^{k-1} \varepsilon_{i}+\left(\varepsilon_{k}-1\right) / 2} .
\end{aligned}
$$

Before reaching the final form of $\Delta_{K_{p, q}}$, we need further modification of (4.3).

\section{LEMMA 4.6.}

$$
D(t)=\frac{1}{2}\left(t^{-\mu / 2}+t^{\mu / 2}\right)-\frac{1}{2}\left(t^{-1 / 2}-t^{1 / 2}\right) \sum_{k=1}^{p-1}(-1)^{k} \varepsilon_{k} t^{-v_{k} / 2} .
$$

PROOF. Applying (2) of Lemma 2.2, we have

$$
\begin{aligned}
2 D(t) & =t^{-\mu / 2}+t^{\mu / 2}+(t-1) \sum_{k=1}^{p-1}(-1)^{k} \varepsilon_{k} t^{-\mu / 2+\sum_{i=1}^{k-1} \varepsilon_{i}+\left(\varepsilon_{k}-1\right) / 2} \\
& =t^{-\mu / 2}+t^{\mu / 2}+(t-1) \sum_{k=1}^{p-1}(-1)^{k} \varepsilon_{k} t^{-\nu_{k} / 2-1 / 2} \\
& =t^{-\mu / 2}+t^{\mu / 2}-\left(t^{-1 / 2}-t^{1 / 2}\right) \sum_{k=1}^{p-1}(-1)^{k} \varepsilon_{k} t^{-\nu_{k} / 2}
\end{aligned}
$$

This implies the lemma.

Now we are ready to prove (1) of Theorem 1.2 .

ProOF OF (1) OF THEOREM 1.2. From (4.4) and the fact that $D(t)=D\left(t^{-1}\right)$, we have

$$
4 D(t)=2\left(t^{-\mu / 2}+t^{\mu / 2}\right)-\left(t^{-1 / 2}-t^{1 / 2}\right) \sum_{k=1}^{p-1}(-1)^{k} \varepsilon_{k}\left(t^{-v_{k} / 2}-t^{\nu_{k} / 2}\right) .
$$

This gives us a more symmetric form (1.3) of $\Delta_{K_{p . q}}$ completing the proof. 


\section{Lucas polynomials}

In this section we study Lucas polynomials. These polynomials are used to transform the normalized Alexander polynomials to the Conway polynomials.

Let $z=t^{-1 / 2}-t^{1 / 2}$ and $V_{n}=\left(t^{-1 / 2}\right)^{n}+\left(-t^{1 / 2}\right)^{n}$. Then $V_{n}$ is expressed as a polynomial in $z$, say $V_{n}(z)$, as follows:

$$
V_{n}(z)=\sum_{j=0}^{[n / 2]} \frac{n}{n-j}\left(\begin{array}{c}
n-j \\
j
\end{array}\right) z^{n-2 j} \quad(n \geq 1) .
$$

These polynomials are called Lucas polynomials and are characterized by

$$
V_{n}(z)=z V_{n-1}(z)+V_{n-2}(z) \quad\left(V_{0}(z)=2, V_{1}(z)=z\right) .
$$

The reader can refer to, for example, [5] for basic properties of Lucas polynomials. From (5.1) we obtain the following lemma.

LEMMA 5.1. Suppose that $z=t^{-1 / 2}-t^{1 / 2}$. Then the following identities hold:

(1) $\left(t^{-1 / 2}\right)^{2 k+1}+\left(-t^{1 / 2}\right)^{2 k+1}=\sum_{j=0}^{k} \frac{2 k+1}{k+1+j}\left(\begin{array}{c}k+1+j \\ k-j\end{array}\right) z^{2 j+1} \quad(k \geq 0)$.

(2) $\left(t^{-1 / 2}\right)^{2 k}+\left(-t^{1 / 2}\right)^{2 k}=\sum_{j=0}^{k} \frac{2 k}{k+j}\left(\begin{array}{l}k+j \\ k-j\end{array}\right) z^{2 j} \quad(k \geq 0)$.

Here we regard $(2 k /(k+j))\left(\begin{array}{l}k+j \\ k-j\end{array}\right)=2$ when $k=j=0$.

The following identities are obtained easily from Lemma 5.1. These formulae are not used in this paper; however, they are of interest on their own right.

COROLLARY 5.2. Let $\Delta_{K}(t)=\sum_{k=0}^{n} a_{k}\left(t^{-k}+t^{k}\right)$ be the Alexander polynomial of a knot $K$. Then the Conway polynomial $\nabla_{K}(z)$ of $K$ is

$$
\nabla_{K}(z)=\sum_{k=0}^{n} a_{k} \sum_{j=0}^{k} \frac{2 k}{k+j}\left(\begin{array}{l}
k+j \\
k-j
\end{array}\right) z^{2 j}
$$

Now we obtain Conway polynomial of a two-bridge knot.

Proof OF (1) OF THeOREM 1.3. Suppose that $z=t^{-1 / 2}-t^{1 / 2}$. Seeing that $\mu$ is even and $v_{k}$ is odd (since $p$ is odd), we have

$$
D(t)=\frac{1}{2}\left(t^{-\mu / 2}+t^{\mu / 2}\right)-\frac{1}{4}\left(t^{-1 / 2}-t^{1 / 2}\right) \sum_{k=1}^{p-1}(-1)^{k} \varepsilon_{k}\left(t^{-v_{k} / 2}-t^{\nu_{k} / 2}\right)
$$




$$
\begin{aligned}
= & \frac{1}{2}\left(t^{-\mu / 2}+t^{\mu / 2}\right)-\frac{1}{4}\left(t^{-1 / 2}-t^{1 / 2}\right) \sum_{k=1}^{p-1}(-1)^{k} \varepsilon_{k} \operatorname{sgn}\left(v_{k}\right)\left(t^{-\left|\nu_{k}\right| / 2}-t^{\left|\nu_{k}\right| / 2}\right) \\
= & \frac{1}{2} \sum_{j=0}^{|\mu| / 2} \frac{|\mu|}{|\mu| / 2+j}\left(\begin{array}{c}
|\mu| / 2+j \\
|\mu| / 2-j
\end{array}\right) z^{2 j} \\
& -\frac{1}{4} z \sum_{k=1}^{p-1}(-1)^{k} \varepsilon_{k} \operatorname{sgn}\left(\nu_{k}\right) \sum_{j=0}^{\left(\left|\nu_{k}\right|-1\right) / 2} \frac{\left|\nu_{k}\right|}{\left|\nu_{k}\right| / 2+1 / 2+j}\left(\begin{array}{c}
\left|\nu_{k}\right| / 2+1 / 2+j \\
\left|\nu_{k}\right| / 2-1 / 2-j
\end{array}\right) z^{2 j+1}
\end{aligned}
$$

(by Lemma 5.1)

$$
\begin{aligned}
= & \frac{1}{2} \sum_{j=0}^{|\mu| / 2} \frac{|\mu|}{|\mu| / 2+j}\left(\begin{array}{c}
|\mu| / 2+j \\
|\mu| / 2-j
\end{array}\right) z^{2 j} \\
& -\frac{1}{4} \sum_{k=1}^{p-1}(-1)^{k} \varepsilon_{k} \sum_{j=0}^{\left(\left|\nu_{k}\right|-1\right) / 2} \frac{\nu_{k}}{\left|\nu_{k}\right| / 2+1 / 2+j}\left(\begin{array}{c}
\left|\nu_{k}\right| / 2+1 / 2+j \\
\left|\nu_{k}\right| / 2-1 / 2-j
\end{array}\right) z^{2 j+2} .
\end{aligned}
$$

This implies (1.5) completing the proof.

To describe the coefficients of the Conway polynomials, we need the following two lemmas. These lemmas can be shown by direct computation, and proofs are left to the reader.

LEMMA 5.3. Let $\mu$ and $j$ be integers such that $j>0$. Then

$$
\frac{|\mu|}{|\mu| / 2+j}\left(\begin{array}{l}
|\mu| / 2+j \\
|\mu| / 2-j
\end{array}\right)=\frac{1}{2^{2 j-1}(2 j) !} \prod_{m=0}^{j-1}\left\{\mu^{2}-(2 m)^{2}\right\}
$$

LEMMA 5.4. Let $v$ and $j$ be integers such that $j>0$. Then

$$
\frac{\nu}{|v| / 2+1 / 2+j}\left(\begin{array}{c}
|\nu| / 2+1 / 2+j \\
|v| / 2-1 / 2-j
\end{array}\right)=\frac{\nu}{2^{2 j}(2 j+1) !} \prod_{m=1}^{j}\left\{v^{2}-(2 m-1)^{2}\right\} \text {. }
$$

Finally, we obtain the coefficients of the Conway polynomial of a two-bridge knot.

PROOF OF (1) OF THEOREM 1.4. From (1.5) and Lemmas 5.3 and 5.4, we have

$$
\begin{aligned}
\nabla_{K_{p, q}}= & \frac{1}{2} \sum_{j=0}^{|\mu| / 2} \frac{|\mu|}{|\mu| / 2+j}\left(\begin{array}{c}
|\mu| / 2+j \\
|\mu| / 2-j
\end{array}\right) z^{2 j} \\
& -\frac{1}{4} \sum_{k=1}^{p-1}(-1)^{k} \varepsilon_{k} \sum_{j=0}^{\left(\left|\nu_{k}\right|-1\right) / 2} \frac{v_{k}}{\left|\nu_{k}\right| / 2+1 / 2+j}\left(\begin{array}{c}
\left|v_{k}\right| / 2+1 / 2+j \\
\left|\nu_{k}\right| / 2-1 / 2-j
\end{array}\right) z^{2 j+2}
\end{aligned}
$$




$$
\begin{aligned}
= & \frac{1}{2} \sum_{j=0}^{|\mu| / 2} \frac{1}{2^{2 j-1}(2 j) !} \prod_{m=0}^{j-1}\left\{\mu^{2}-(2 m)^{2}\right\} z^{2 j} \\
& -\frac{1}{4} \sum_{k=1}^{p-1}(-1)^{k} \varepsilon_{k} \sum_{j=0}^{\left(\left|\nu_{k}\right|-1\right) / 2} \frac{v_{k}}{2^{2 j}(2 j+1) !} \prod_{m=1}^{j}\left\{v_{k}^{2}-(2 m-1)^{2}\right\} z^{2 j+2} .
\end{aligned}
$$

Taking the $(2 j+2)$ th coefficient in the last expression, we obtain Theorem $1.4(1)$.

\section{Polynomials of a two-bridge link $K_{p, q}$}

In this section we assume that $p$ is even, in which case $K_{p, q}$ is not a knot but a two-component link. We will describe the Alexander and Conway polynomials. More precisely, we give proofs of (2) of Theorem 1.2, Theorem 1.3 and Theorem 1.4. We set

$$
E_{1}(t):=\left(\frac{\partial R_{2}}{\partial S_{1}}\right)^{\alpha} \quad \text { and } \quad E_{2}(t):=\left(\frac{\partial R_{2}}{\partial S_{2}}\right)^{\alpha}
$$

Then both $E_{1}(t)$ and $E_{2}(t)$ are reduced Alexander polynomials for the two-bridge link $K_{p, q}$. They are calculated as:

LEMMA 6.1. (1) $E_{1}(t)=1-t^{\mu}+(t-1) \sum_{k=1, k \text { even }}^{p-1} \varepsilon_{k} t^{\sum_{i=1}^{k-1} \varepsilon_{i}+\left(\varepsilon_{k}-1\right) / 2}$.

(2) $E_{2}(t)=(t-1) \sum_{k=1, k \text { odd }}^{p-1} \varepsilon_{k} t^{\sum_{i=1}^{k-1} \varepsilon_{i}+\left(\varepsilon_{k}-1\right) / 2}$.

PROOF. We take the free derivative $\partial / \partial S_{1}$ of $L_{2}$ and then abelianize it:

$$
\begin{aligned}
\left(\frac{\partial L_{2}}{\partial S_{1}}\right)^{\alpha} & =\left(\frac{\partial S_{2}^{\varepsilon_{1}} S_{1}^{\varepsilon_{2}} \cdots S_{1}^{\varepsilon_{p-2}} S_{2}^{\varepsilon_{p-1}}}{\partial S_{1}}\right)^{\alpha} \\
& =\left(\sum_{k=1, k \text { even }}^{p-1} \varepsilon_{k} S_{2}^{\varepsilon_{1}} S_{1}^{\varepsilon_{2}} \cdots S_{1}^{\left(\varepsilon_{k}-1\right) / 2}\right)^{\alpha} \\
& =\sum_{k=1, k \text { even }}^{p-1} \varepsilon_{k} t^{\sum_{i=1}^{k-1} \varepsilon_{i}+\left(\varepsilon_{k}-1\right) / 2}
\end{aligned}
$$

Similarly, we have

$$
\left(\frac{\partial L_{2}}{\partial S_{2}}\right)^{\alpha}=\sum_{k=1, k \text { odd }}^{p-1} \varepsilon_{k} t^{\sum_{i=1}^{k-1} \varepsilon_{i}+\left(\varepsilon_{k}-1\right) / 2}
$$


Next we calculate $E_{1}(t)=\left(\partial R_{2} / \partial S_{1}\right)^{\alpha}$ as follows:

$$
\begin{aligned}
E_{1}(t) & =\left(\frac{\partial S_{1} L_{2} S_{1}^{-1} L_{2}^{-1}}{\partial S_{1}}\right)^{\alpha} \\
& =1+\left(S_{1} \frac{\partial L_{2}}{\partial S_{1}}\right)^{\alpha}-\left(S_{1} L_{2} S_{1}^{-1}\right)^{\alpha}-\left(S_{1} L_{2} S_{1}^{-1} L_{2}^{-1} \frac{\partial L_{2}}{\partial S_{1}}\right)^{\alpha} \\
& =1-t^{\sum_{i=1}^{p-1} \varepsilon_{i}}+(t-1)\left(\frac{\partial L_{2}}{\partial S_{1}}\right)^{\alpha} \\
& =1-t^{\mu}+(t-1) \sum_{k=1, k \text { even }}^{p-1} \varepsilon_{k} t^{\sum_{i=1}^{k-1} \varepsilon_{i}+\left(\varepsilon_{k}-1\right) / 2} .
\end{aligned}
$$

Similarly, we have

$$
\begin{aligned}
E_{2}(t) & =\left(\frac{\partial S_{1} L_{2} S_{1}^{-1} L_{2}^{-1}}{\partial S_{2}}\right)^{\alpha}=\left(S_{1} \frac{\partial L_{2}}{\partial S_{2}}\right)^{\alpha}-\left(S_{1} L_{2} S_{1}^{-1} L_{2}^{-1} \frac{\partial L_{2}}{\partial S_{2}}\right)^{\alpha} \\
& =(t-1)\left(\frac{\partial L_{2}}{\partial S_{2}}\right)^{\alpha}=(t-1) \sum_{k=1, k \text { odd }}^{p-1} \varepsilon_{k} t^{\sum_{i=1}^{k-1} \varepsilon_{i}+\left(\varepsilon_{k}-1\right) / 2} .
\end{aligned}
$$

This completes the proof.

Now we need two lemmas which show fundamental properties of $E_{1}(t)$ and $E_{2}(t)$.

LEMMA 6.2. $E_{1}(t)=-E_{2}(t)$.

PROOF. The proof is the same as that of Lemma 4.2.

Next we study another property of $E_{2}(t)$.

LEMMA 6.3. $E_{2}(t)=-t^{\mu} E_{2}\left(t^{-1}\right)$.

PROOF. Recall that

$$
E_{2}(t)=(t-1) \sum_{k=1, k \text { odd }}^{p-1} \varepsilon_{k} t^{\sum_{i=1}^{k-1} \varepsilon_{i}+\left(\varepsilon_{k}-1\right) / 2}
$$

Then we have

$$
\begin{aligned}
E_{2}(t) & =(t-1) \sum_{k=1, k \text { odd }}^{p-1} \varepsilon_{k} t^{\sum_{i=1}^{k-1} \varepsilon_{i}+\left(\varepsilon_{k}-1\right) / 2} \\
& =(t-1) \sum_{k=1, k \text { odd }}^{p-1} \varepsilon_{k} t^{\mu-\sum_{i=1}^{p-k} \varepsilon_{i}+\left(\varepsilon_{k}-1\right) / 2} \quad \text { (by Lemma 2.2 (1)) }
\end{aligned}
$$




$$
\begin{aligned}
& =(t-1) \sum_{k=1, k \text { odd }}^{p-1} \varepsilon_{p-k} t^{\mu-\sum_{i=1}^{p-k} \varepsilon_{i}+\left(\varepsilon_{p-k}-1\right) / 2} \quad \text { (by Lemma 2.1 (1)) } \\
& =(t-1) \sum_{k=1, k \text { odd }}^{p-1} \varepsilon_{k} t^{\mu-\sum_{i=1}^{k} \varepsilon_{i}+\left(\varepsilon_{k}-1\right) / 2} \quad \text { (note that } p \text { is even) } \\
& =t^{\mu}\left\{(t-1) \sum_{k=1, k \text { odd }}^{p-1} \varepsilon_{k} t^{-\sum_{i=1}^{k} \varepsilon_{i}+\varepsilon_{k} / 2-1 / 2}\right\} \\
& =t^{\mu}\left\{\left(1-t^{-1}\right) \sum_{k=1, k \text { odd }}^{p-1} \varepsilon_{k} t^{-\sum_{i=1}^{k-1} \varepsilon_{i}-\left(\varepsilon_{k}-1\right) / 2}\right\} \\
& =t^{\mu}\left\{-E_{2}\left(t^{-1}\right)\right\}=-t^{\mu} E_{2}\left(t^{-1}\right) .
\end{aligned}
$$

This completes the proof.

Now we are ready to get the normalized Alexander polynomial for $K_{p, q}$. We put $E(t)=-t^{-\mu / 2} E_{2}(t)$.

LEMMA 6.4. $E(t)$ is the normalized Alexander polynomial. Namely, $E(t)$ satisfies

$$
E\left(t^{-1}\right)=-E(t) \quad \text { and } \quad \lim _{t \rightarrow 1} \frac{E(t)}{t^{-1 / 2}-t^{1 / 2}}=l k\left(K_{p, q}\right)
$$

where $l k\left(K_{p, q}\right)$ is the linking number of the oriented two-component link $K_{p, q}$.

PROOF. We have

$$
E\left(t^{-1}\right)=-t^{\mu / 2} E_{2}\left(t^{-1}\right)=-t^{\mu / 2}\left\{-t^{-\mu} E_{2}(t)\right\}=t^{-\mu / 2} E_{2}(t)=-E(t) .
$$

Furthermore, we have

$$
\begin{aligned}
\lim _{t \rightarrow 1} \frac{E(t)}{t^{-1 / 2}-t^{1 / 2}} & =\lim _{t \rightarrow 1} \frac{-t^{-\mu / 2}(t-1)}{t^{-1 / 2}-t^{1 / 2}} \sum_{k=1, k \text { odd }}^{p-1} \varepsilon_{k} t^{\sum_{i=1}^{k-1} \varepsilon_{i}+\left(\varepsilon_{k}-1\right) / 2} \\
& =\lim _{t \rightarrow 1} t^{-\mu / 2} \sum_{k=1, k \text { odd }}^{p-1} \varepsilon_{k} t^{\sum_{i=1}^{k-1} \varepsilon_{i}+\varepsilon_{k} / 2}=\sum_{k=1, k \text { odd }}^{p-1} \varepsilon_{k} .
\end{aligned}
$$

Then we can easily identify the last term $\sum_{k=1, k \text { odd }}^{p-1} \varepsilon_{k}$ first with $\sum_{k=1}^{p / 2} \varepsilon_{2 k-1}$, and then with $l k\left(K_{p, q}\right)$ (refer to [3, page 185]). These imply that $E(t)$ is normalized.

The following form of $E(t)$ is also useful. 
LEMMA 6.5.

$$
E(t)=\frac{1}{2}\left(t^{-\mu / 2}-t^{\mu / 2}\right)-\frac{1}{2}\left(t^{-1 / 2}-t^{1 / 2}\right) \sum_{k=1}^{p-1}(-1)^{k} \varepsilon_{k} t^{-v_{k} / 2} .
$$

ProOF.

$$
\begin{aligned}
2 E(t)= & -t^{-\mu / 2} E_{2}(t)+t^{-\mu / 2} E_{1}(t) \\
= & -t^{-\mu / 2}(t-1) \sum_{k=1, k \text { odd }}^{p-1} \varepsilon_{k} t^{\sum_{i=1}^{k-1} \varepsilon_{i}+\left(\varepsilon_{k}-1\right) / 2}+t^{-\mu / 2}\left(1-t^{\mu}\right) \\
& +t^{-\mu / 2}(t-1) \sum_{k=1, k \text { even }}^{p-1} \varepsilon_{k} t^{\sum_{i=1}^{k-1} \varepsilon_{i}+\left(\varepsilon_{k}-1\right) / 2} \\
= & t^{-\mu / 2}\left(1-t^{\mu}\right)+t^{-\mu / 2}(t-1) \sum_{k=1}^{p-1}(-1)^{k} \varepsilon_{k} t^{t_{i=1}^{k-1} \varepsilon_{i}+\left(\varepsilon_{k}-1\right) / 2} \\
= & \left.t^{-\mu / 2}-t^{\mu / 2}+(t-1) \sum_{k=1}^{p-1}(-1)^{k} \varepsilon_{k} t^{-v_{k} / 2-1 / 2} \quad \text { (by Lemma } 2.2(2)\right) \\
= & t^{-\mu / 2}-t^{\mu / 2}-\left(t^{-1 / 2}-t^{1 / 2}\right) \sum_{k=1}^{p-1}(-1)^{k} \varepsilon_{k} t^{-v_{k} / 2} .
\end{aligned}
$$

Now we are ready to prove (2) of Theorem 1.2.

ProOf OF (2) OF THEOREM 1.2. From (6.1) and the fact that $E(t)=-E\left(t^{-1}\right)$, we have

$$
2 E(t)=\left(t^{-\mu / 2}-t^{\mu / 2}\right)-\frac{1}{2}\left(t^{-1 / 2}-t^{1 / 2}\right) \sum_{k=1}^{p-1}(-1)^{k} \varepsilon_{k}\left(t^{-v_{k} / 2}+t^{\nu_{k} / 2}\right) .
$$

This implies (1.4).

Next we obtain the Conway polynomial of a two-bridge link.

PROOF OF (2) OF THEOREM 1.3. Suppose that $z=t^{-1 / 2}-t^{1 / 2}$. Observing that $\mu$ is odd and $\nu_{k}$ is even (since $p$ is even), we have

$$
\begin{aligned}
E(t) & =\frac{1}{2}\left(t^{-\mu / 2}-t^{\mu / 2}\right)-\frac{1}{4}\left(t^{-1 / 2}-t^{1 / 2}\right) \sum_{k=1}^{p-1}(-1)^{k} \varepsilon_{k}\left(t^{-\nu_{k} / 2}+t^{\nu_{k} / 2}\right) \\
& =\frac{1}{2} \operatorname{sgn}(\mu)\left(t^{-|\mu| / 2}-t^{\mid \mu / 2}\right)-\frac{1}{4}\left(t^{-1 / 2}-t^{1 / 2}\right) \sum_{k=1}^{p-1}(-1)^{k} \varepsilon_{k}\left(t^{-\left|\nu_{k}\right| / 2}+t^{\left|\nu_{k}\right| / 2}\right)
\end{aligned}
$$




$$
\begin{aligned}
= & \frac{1}{2} \operatorname{sgn}(\mu) \sum_{j=0}^{(|\mu|-1) / 2} \frac{|\mu|}{|\mu| / 2+1 / 2+j}\left(\begin{array}{c}
|\mu| / 2+1 / 2+j \\
|\mu| / 2-1 / 2-j
\end{array}\right) z^{2 j+1} \\
& -\frac{1}{4} z \sum_{k=1}^{p-1}(-1)^{k} \varepsilon_{k} \sum_{j=0}^{\left|\nu_{k}\right| / 2} \frac{\left|\nu_{k}\right|}{\left|\nu_{k}\right| / 2+j}\left(\begin{array}{c}
\left|\nu_{k}\right| / 2+j \\
\left|\nu_{k}\right| / 2-j
\end{array}\right) z^{2 j} \\
= & \frac{1}{2} \sum_{j=0}^{(|\mu|-1) / 2} \frac{\mu}{|\mu| / 2+1 / 2+j}\left(\begin{array}{c}
|\mu| / 2+1 / 2+j \\
|\mu| / 2-1 / 2-j
\end{array}\right) z^{2 j+1} \\
& -\frac{1}{4} \sum_{k=1}^{p-1}(-1)^{k} \varepsilon_{k} \sum_{j=0}^{\left|\nu_{k}\right| / 2} \frac{\left|\nu_{k}\right|}{\left|\nu_{k}\right| / 2+j}\left(\begin{array}{c}
\left|v_{k}\right| / 2+j \\
\left|v_{k}\right| / 2-j
\end{array}\right) z^{2 j+1}
\end{aligned}
$$

This implies (1.6).

Finally, we prove (2) of Theorem 1.4.

Proof of (2) OF THEOREM 1.4. Suppose that $z=t^{-1 / 2}-t^{1 / 2}$. Then we have,

$$
\begin{aligned}
\nabla_{K_{p, q}}= & \frac{1}{2} \sum_{j=0}^{(|\mu|-1) / 2} \frac{\mu}{|\mu| / 2+1 / 2+j}\left(\begin{array}{c}
|\mu| / 2+1 / 2+j \\
|\mu| / 2-1 / 2-j
\end{array}\right) z^{2 j+1} \\
& -\frac{1}{4} \sum_{k=1}^{p-1}(-1)^{k} \varepsilon_{k} \sum_{j=0}^{\left|\nu_{k}\right| / 2} \frac{\left|v_{k}\right|}{\left|\nu_{k}\right| / 2+j}\left(\begin{array}{c}
\left|\nu_{k}\right| / 2+j \\
\left|v_{k}\right| / 2-j
\end{array}\right) z^{2 j+1} \\
= & \frac{1}{2} \sum_{j=0}^{(|\mu|-1) / 2} \frac{\mu}{2^{2 j}(2 j+1) !} \prod_{m=1}^{j}\left\{\mu^{2}-(2 m-1)^{2}\right\} z^{2 j+1} \\
& -\frac{1}{4} \sum_{k=1}^{p-1}(-1)^{k} \varepsilon_{k} \sum_{j=0}^{\left|v_{k}\right| / 2} \frac{1}{2^{2 j-1}(2 j) !} \prod_{m=0}^{j-1}\left\{v_{k}^{2}-(2 m)^{2}\right\} z^{2 j+1}
\end{aligned}
$$

by Lemmas 5.3 and 5.4 . Taking the $(2 j+1)$ th coefficient in the last expression, we obtain (2) of Theorem 1.4.

\section{References}

[1] B. C. Berndt and R. J. Evans, 'On Rademacher's multiplier system for the classical theta-function', Contemp. Math. 166 (1994), 1-7.

[2] J. S. Birman, Braids, links, and mapping class groups, Ann. Math. Studies 82 (Princeton University Press, Princeton, 1975).

[3] G. Burde and H. Zieschang, Knot, Studies in Math. 5 (Walter de Gruyter, 1986).

[4] R. H. Crowell and R. H. Fox, Introduction to knot theory (Springer, New York, 1977). 
[5] P. Filipponi and A. F. Horadam, 'Derivative sequences of Fibonacci and Lucas polynomials', in: Applications of Fibonacci numbers, Vol. 4 (ed. G. E. Bergum et al.) (Kluwer, Dordrecht, 1991) pp. 99-108.

[6] S. Fukuhara, 'Modular forms, generalized Dedekind symbols and period polynomials', Math. Ann. 310 (1998), 83-101.

[7] R. I. Hartley, 'On two-bridged knot polynomials', J. Austral. Math. Soc. 28 (1979), 241-249.

[8] T. Kanenobu, 'Alexander polynomials of two-bridge links', J. Austral. Math. Soc. 36 (1984), $59-68$.

[9] A. Kawauchi, A survey of knot theory (Birkhäuser, Basel, 1996).

[10] R. Tuler, 'On the linking number of a 2-bridge link', Bull. London Math. Soc. 13 (1981), 540-544.

Department of Mathematics

Tsuda College

Tsuda-machi 2-1-1

Kodaira-shi

Tokyo 187-8577

Japan

e-mail: fukuhara@tsuda.ac.jp 